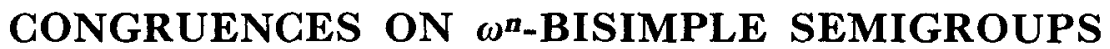

\author{
R. J. WARNE
}

(Received 19 September 1966)

Let $S$ be a bisimple semigroup and let $E_{S}$ denote its set of idempotents. We may partially order $E_{S}$ in the following manner: if $e, f \in E_{S}, e \leqq f$ if and only if $e f=f e=e$. We then say that $E_{S}$ is under or assumes its natural order. Let $I^{0}$ denote the non-negative integers and let $n$ denote a natural number. If $E_{S}$, under its natural order, is order isomorphic to $\left(I^{0}\right)^{n}$ under the reverse of the usual lexicographic order, we call $S$ an $\omega^{n}$-bisimple semigroup ${ }^{1}$. (See $[\mathbf{9}]$ for an explanation of notation.) We determined the structure of $\omega^{n}$-bisimple semigroups completely mod groups in [9]. The $\omega^{n}$-bisimple semigroups, the $I$-bisimple semigroups [8], and the $\omega^{n} I-$ bisimple semigroups [9] are classes of simple semigroups except completely simple semigroups whose structure has been determined mod groups.

The purpose of this paper is to give a complete determination of the congruence relations of an $\omega^{n}$-bisimple semigroup mod the congruences of groups. We show that if $\rho$ is a congruence relation on an $\omega^{n}$-bisimple semigroup $S, \rho$ is a group congruence ( $S / \rho$ is a group), $\rho$ is an idempotent separating congruence (each $\rho$-class contains at most one idempotent), or $\rho$ is an $\omega^{k}$-bisimple congruence ( $S / \rho$ is an $\omega^{k}$-bisimple semigroup) for some $k$ with $1 \leqq k \leqq n-1$. To determine the group congruences, we give an explicit determination of the maximal group homomorphic image $H$ of $S$ including the defining homomorphism. Clearly, the group congruences are in a $1-\mathbf{l}$ correspondence with the normal subgroups of $H$. We determine the idempotent separating congruences and the $\omega^{k}$-bisimple congruences in terms of certain normal subgroups of the group of units of $S$.

We say that $\left(I^{0}\right)^{n}$ is under the reverse of the usual lexicographic order if $\left(a_{1}, a_{2}, \cdots, a_{n}\right)<\left(b_{1}, b_{2}, \cdots, b_{n}\right)$ if and only if $a_{1}>b_{1}$, or $a_{1}=b_{1}$ and $a_{2}>b_{2}$, or $\cdots a_{i}=b_{i}$ for $1 \leqq i<k$ and $a_{k}>b_{k}$, or $\cdots, a_{i}=b_{i}$ for $1 \leqq i<n$ and $a_{n}>b_{n}$.

Unless otherwise specified we will use the definitions and terminology of $[2]$.

1 Change in notation: In [6, 11], we called an $\omega^{n}$-bisimple semigroup an $L_{n}$-bisimple semigroup. For simplicity and to utilize standard notation, we finally adopted the present notation. 


\section{The nature of the congruences}

In this section, we show that if $\rho$ is a congruence relation on an $\omega^{n}$ bisimple semigroup, $\rho$ is a group congruence, $\rho$ is an idempotent separating congruence, or $\rho$ is an $\omega^{k}$-bisimple congruence for some $k$ with $1 \leqq k \leqq n-1$.

Let us first give an indication of the structure theorems on which our development is based.

Let $C_{1}$ be the bicyclic semigroup and let $T$ be a semigroup. Let $C_{1} \circ T=C_{1} \times T$ under the multiplication

$$
((n, m), s)((p, q), t)=((n, m)(p, q), f(m, p))
$$

where $f(m, p)=s, t$, or $s t$ according to whether $m>p, p>m$, or $p=m$ and where juxtaposition denotes multiplication in $C_{1}$ and $T$. We define $C_{2}=C_{1} \circ C_{1}, C_{3}=C_{1} \circ C_{2}, \cdots, C_{n}=C_{1} \circ C_{n-1}$. We call $C_{n}$ the $2 n$-cyclic semigroup [6]. The structure of an $\omega^{n}$-bisimple $S$ is described completely in terms of a group $G$ (the group of units of $S$ ), endomorphisms $\gamma_{1}, \gamma_{2}, \cdots, \gamma_{n}$ of $G$, elements $t_{1}, t_{2}, \cdots, t_{\varphi(n)}(\varphi(n)=n(n-1) / 2)$ of $G$, and $C_{n}$. Thus, if $S$ is an $\omega^{n}$-bisimple semigroup, we will write

$$
S=\left(G, \gamma_{1}, \gamma_{2}, \cdots, \gamma_{n}, t_{1}, t_{2}, \cdots, t_{p(n)}\right) .
$$

In fact, $S \cong G \times C_{n}$ under a suitable multiplication and conversely, $\left[9\right.$, theorem 2.3]. An $\omega^{n}$-bisimple semigroup $S$ is a bisimple inverse semigroup with identity with

$$
E_{S}=\left(\left(e,\left(a_{1}, a_{1}\right), \cdots,\left(a_{n}, a_{n}\right)\right): a_{i} \in I^{0}, \quad 1 \leqq i \leqq n\right)
$$

where $e$ is the identity of $G$. The inverse of $\left(g,\left(a_{1}, b_{1}\right),\left(a_{2}, b_{2}\right), \cdots,\left(a_{n}, b_{n}\right)\right)$ is $\left(g^{-1},\left(b_{1}, a_{1}\right),\left(b_{2}, a_{2}\right), \cdots,\left(b_{n}, a_{n}\right)\right)$ where $g^{-1}$ is the inverse of $g$ in the group $G$. Let $\mathscr{R}, \mathscr{L}$, and $\mathscr{H}$ denote Green's relations [2]. We note that

$$
\left(g,\left(a_{1}, b_{1}\right),\left(a_{2}, b_{2}\right), \cdots,\left(a_{n}, b_{n}\right)\right) \mathscr{R}\left(h,\left(c_{1}, d_{1}\right),\left(c_{2}, d_{2}\right), \cdots,\left(c_{n}, d_{n}\right)\right)
$$

if and only if $b_{i}=d_{i}$ for $1 \leqq i \leqq n$. Hence,

$$
\left(g,\left(a_{1}, b_{1}\right), \cdots,\left(a_{n}, b_{n}\right)\right) \mathscr{H}\left(h,\left(c_{1}, d_{1}\right), \cdots,\left(c_{n}, d_{n}\right)\right)
$$

if and only if $a_{i}=c_{i}$ and $b_{i}=d_{i}$ for $1 \leqq i \leqq n$. These facts follow from $[9$, theorem 2.3].

We will also utilize a structure theorem for a class of right cancellative semigroups. Let $P$ be a right cancellative semigroup with identity. The partially ordered system of principal left ideals of $P$ ordered by inclusion is termed the ideal structure of $P$. If the ideal structure of $P$ is order isomorphic to $\left(I^{0}\right)^{n}$ under the reverse of the usual lexicographic order, $P$ is called an $\omega^{n}$-right cancellative semigroup. The structure of an $\omega^{n}$-right 
cancellative semigroup $P$ is described completely in terms of a group $G$ (the group of units of $P$ ), endomorphisms $\gamma_{1}, \gamma_{2}, \cdots, \gamma_{n}$ of $G$, elements $t_{1}, t_{2}, \cdots, t_{\varphi(n)}$ of $G$, and $\left(I^{0}\right)^{n}$. Thus, we write

$$
P=\left(G,\left(I^{0}\right)^{n}, \gamma_{1}, \gamma_{2}, \cdots, \gamma_{n}, t_{1}, t_{2}, \cdots, t_{\varphi(n)}\right) .
$$

In fact, $P$ is an $\omega^{n}$-right cancellative semigroup if and only if $P \cong G x\left(I^{0}\right)^{n}$, where $G$ is a group, under a suitable multiplication [9, theorem 1.4]. If $G$ is a group and $y \in G$, we define $x C_{y}=y x y^{-1}$ for all $x \in G$.

The following lemma which is based on the homomorphism theory of [5] will be utilized in this section and in section 4 .

LemMa 1.1. Let

and

$$
S=\left(G, \gamma_{1}, \gamma_{2}, \cdots, \gamma_{n}, w_{1}, w_{2}, \cdots, w_{\varphi(n)}\right)
$$

$$
S^{*}=\left(G^{*}, \alpha_{1}, \alpha_{2}, \cdots, \alpha_{k}, t_{1}, t_{2}, \cdots, t_{\varphi(k)}\right)
$$

be $\omega^{n}$ and $\omega^{k}$-bisimple semigroups respectively with $n>k$. Let $\theta$ be a homomorphism of $S$ onto $S^{*}$. Then there exists a homomorphism $f$ of $G$ onto $G^{*}$ and elements $z_{1}, z_{2}, \cdots, z_{n}$ of $G^{*}$ such that

$$
\begin{aligned}
& z_{l+s} \alpha_{l} t_{\varphi(k-l)+s} C_{z_{l}}=w_{\varphi(n-l)+s} t \quad \text { if } \quad 1 \leqq l<k, 1 \leqq s \leqq k-l . \\
& z_{l+s} \alpha_{l} C_{z_{l}}=w_{\varphi(n-l)+s} f \quad \text { if } \quad k-l+1 \leqq s \leqq n-l, 1 \leqq l \leqq k . \\
& z_{l+s} C_{z_{l}}=w_{q(n-l)+s} f \text { if } k<l<n, 1 \leqq s \leqq n-l \text {. } \\
& f \alpha_{i} C_{z_{i}}=\gamma_{i} f \quad \text { if } \quad 1 \leqq i \leqq k \text {. } \\
& f C_{z_{i}}=\gamma_{i} f \quad \text { if } \quad 1+k \leqq i \leqq n \text {. }
\end{aligned}
$$

For each

$$
\begin{aligned}
\left(g,\left(a_{1}, b_{1}\right),\right. & \left.\left(a_{2}, b_{2}\right), \cdots,\left(a_{n}, b_{n}\right)\right) \in S,\left(g,\left(a_{1}, b_{1}\right),\left(a_{2}, b_{2}\right), \cdots,\left(a_{n}, b_{n}\right)\right) \theta \\
= & \left(\left(\prod_{a_{1}-1}^{j=0} z_{1}^{-1} \alpha_{1}^{j} \alpha_{2}^{a_{2}} \cdots \alpha_{k}^{a_{k}}\right) \cdots\left(\prod_{a_{k-1}-1}^{j=0} z_{k-1}^{-1} \alpha_{k-1}^{j} \alpha_{k}^{a_{k}}\right)\right. \\
& \cdot\left(\prod_{a_{k}-1}^{j=0} z_{k}^{-1} \alpha_{k}^{j}\right) z_{k+1}^{-a_{k+1}} \cdots z_{n}^{-a_{n}} g f \cdot z_{n}^{b_{n}} \cdots z_{k+1}^{b_{k+1}}\left(\prod_{j=0}^{b_{k}-1} z_{k} \alpha_{k}^{j}\right) \\
& \left.\left.\cdot\left(\prod_{j=0}^{b_{k-1}-1} z_{k-1} \alpha_{k-1}^{j} \alpha_{k}^{b_{k}}\right) \cdots \prod_{j=0}^{b_{1}-1} z_{1} \alpha_{1}^{j} \alpha_{2}^{b_{2}} \cdots \alpha_{k}^{b_{k}}\right),\left(a_{1}, b_{1}\right), \cdots,\left(a_{k}, b_{k}\right)\right)
\end{aligned}
$$

if $a_{1}, b_{1}, \cdots, a_{k}, b_{k} \geqq 1$. If $a_{j}\left(b_{j}\right)=0$ for some $j$, the corresponding factor is $e^{*}$, the identity of $G^{*}$.

Proof. By [9, theorem 2.3] and [9, theorem 1.4], the right unit subsemigroups of $S$ and $S^{*}$ are $P=\left(G,\left(I^{0}\right)^{n}, \gamma_{1}, \gamma_{2}, \cdots, \gamma_{n}, w_{1}, w_{2}, \cdots, w_{\varphi(n)}\right)$ 
and $P^{*}=\left(G^{*},\left(I^{0}\right)^{k}, \alpha_{1}, \alpha_{2}, \ldots, \alpha_{k}, t_{1}, t_{2}, \cdots, t_{\varphi(k)}\right)$ respectively. If we apply [1, main theorem] to $P=\left(G,\left(I^{0}\right)^{n}, \gamma_{1}, \gamma_{2}, \cdots, \gamma_{n}, w_{1}, w_{2}, \cdots, w_{\varphi(n)}\right)$, we will denote the resulting semigroup by $T$. Since the group of units of $P$ is $\{(g, 0,0, \cdots, 0): g \in G\}$ by $[9$, theorem 1.4], it follows from $[1$, p. 548, equation 1.2] and [9, theorem 1.4] that each element of $T$ may be given the 'normal representation' $\left(\left(e, a_{1}, a_{2}, \cdots, a_{n}\right),\left(g, b_{1}, b_{2}, \cdots, b_{n}\right)\right)$ (notation of $[1$, main theorem $]$ ). We showed in [9, proof of theorem 2.3$]$ that

$$
\left(\left(e, a_{1}, a_{2}, \cdots, a_{n}\right),\left(g, b_{1}, b_{2}, \cdots, b_{n}\right)\right) \delta=\left(g,\left(a_{1}, b_{1}\right),\left(a_{2}, b_{2}\right), \cdots,\left(a_{n}, b_{n}\right)\right)
$$

defines an isomorphism of $T$ onto $S=\left(G, C_{n}, \gamma_{1}, \gamma_{2}, \ldots, \gamma_{n}, w_{1}, w_{2}, \cdots, w_{p(n)}\right)$. Let $\delta^{*}$ denote the corresponding isomorphism of $T^{*}$ onto $S^{*}$. Let $\theta$ be a homomorphism of $S$ onto $S^{*}$. Hence, $M=\delta \theta\left(\delta^{*}\right)^{-1}$ is a homomorphism of $T$ onto $T^{*}$. Thus, $M$ is given by [5, p. 1114, equation 1.14] by virtue of $[5$, p. 1113 , theorem 1.1$]$. Since $M$ maps $T$ onto $T^{*}$, it follows that the $k$ in $\left[5\right.$, p. 1114, equation 1.14] is an element of the group of units of $P^{*}$ by virtue of $[1$, p. 548, equation 1.2]. Hence, by [5, p. 1113, equation 1.13], $k$ may be taken to be the identity of $P^{*}$ (actually, the homomorphism $N$ in [5, equation 1.14] is replaced by $N C_{k^{-1}}$ where $x C_{k^{-1}}=k^{-1} x k$ for $\left.x \in G^{*}\right)$. We will now utilize [5, theorem 2.3] to determine $N$ and to establish (1)-(5). $P$ is a Rédei-Schreier extension [5, p. 1117] of $G$ by $P / \mathscr{L}$ by [5, theorem 2.2] and $[9$, theorem 1.4]. In the notation of [5, p. 1117 and theorem 2.3$]$, the 'factor' and 'endomorphism' terms of this extension are given by [9, equation 1.21 and equation 1.22 respectively (with ' $z$ ' replaced by ' $w$ ')]. These terms may be derived by comparing [5, equation 2.2$]$ with [9, (A) of theorem 1.4]. If we substitute [5, p. 1119, equation 2.14] (with $M$ replaced by $N$ ) into the modified form of [5, equation 1.14] and utilize [1, equation 1.2] to obtain the 'normal' representation, we see that $g$ maps $P / \mathscr{L}$ onto $P^{*} / \mathscr{L}^{*}$. Hence, utilizing $\left[9\right.$, Corollary 1.1], we see that $\left(a_{1}, a_{2}, \cdots, a_{n}\right) g=\left(a_{1}, a_{2}, \cdots, a_{k}\right)$ for all $\left(a_{1}, a_{2}, \cdots, a_{n}\right) \in P / \mathscr{L}$. Let $U_{s}$ be the element' of $\left(I^{0}\right)^{n}$ with ' 1 ' in the $s$-th position and zeroes elsewhere and let $U_{s} h=z_{s}$ (notation of $[5$, theorem 2.3$]$ ). Thus, by substituting [9, equation 1.21 ] and [9, equation 1.22] into [5, p. 1119, equation 2.12$]$ and utilizing [9, corollary 1.1], we obtain $(1)-(3)$. If we substitute [9, equation 1.22$]$ into [5, page 1119 , equation 2.13], we obtain (4) and (5).

Similarly, utilizing $[9$, corollary 1.1$]$, [9, equation 1.21 and equation $1.22]$, and [5, p. 1119 , equation 2.12$]$, we obtain

$$
\begin{aligned}
\left(b_{1}, b_{2}, \cdots, b_{n}\right) h= & z_{n}^{b_{n}} \cdots z_{k+1}^{b_{k+1}}\left(\prod_{j=0}^{b_{k}-1} z_{l_{k}} \alpha_{k}^{j}\right) \\
& \cdot\left(\prod_{j=0}^{b_{k-1}-1} z_{k-1} \alpha_{k-1}^{j} \alpha_{k}^{b_{k}}\right) \cdots\left(\prod_{j=0}^{b_{1}-1} z_{1} \alpha_{1}^{j} \alpha_{2}^{b_{2}} \cdots \alpha_{k}^{b_{k}}\right) .
\end{aligned}
$$

Thus, $N$ is given by $[5$, p. 1119 , equation 2.14$]$. We next substitute $N$ 
into the modified form of [5, equation 1.14] and reduce both sides of the equation to 'normal form' to obtain M. Hence, $\theta=\delta^{-1} M \delta^{*}$.

We utilize the next corollary in the determination of the nature of the congruences of an $\omega^{n}$-bisimple semigroup.

Corollary 1.1. Let $S=\left(G, C_{n}, \gamma_{1}, \gamma_{2}, \cdots, \gamma_{n}, w_{1}, w_{2}, \cdots, w_{\varphi(n)}\right)$ be an $\omega^{n}$-bisimple semigroup and let e be the identity of $G$. Suppose $\rho$ is a congruence relation on $S$. Then, $\rho$ is an $\omega^{k}$-bisimple congruence $(k<n)$ on $S$ if and only if the following condition is satisfied:

$$
\left(e,\left(a_{1}, a_{1}\right), \cdots,\left(a_{n}, a_{n}\right)\right) \rho\left(e,\left(b_{1}, b_{1}\right), \cdots,\left(b_{n}, b_{n}\right)\right)
$$

if and only if $a_{s}=b_{s}$ for $1 \leqq s \leqq k$ for all $a_{i}, b_{i} \in I^{0}$.

Proof. Suppose $\rho$ is an $\omega^{k}$-bisimple congruence on $S$. Thus, the condition is a consequence of lemma 1.1. Conversely, suppose the condition is satisfied. Thus, $\rho$ is an $\omega^{k}$-bisimple congruence on $S$ by $[3$, p. 62 , theorem $8.48]$ and [5, p. 1111].

THEOREM 1.1. Let $S$ be an $\omega^{n}$-bisimple semigroup. Then, each congruence on $S$ is a group congruence, an idempotent separating congruence, or an $\omega^{k}$-bisimple congruence for some $k \in\{1,2, \cdots, n-1\}$.

Proof. Let $\rho$ be a congruence relation on an $\omega^{n}$-bisimple semigroup $S=\left(G, C_{n}, \gamma_{1}, \gamma_{2}, \cdots, \gamma_{n}, t_{1}, t_{2}, \cdots, t_{\varphi(n)}\right)$ which is not an idempotent separating congruence. There exists a smallest non-negative integer $k$ such that for some $a, b \in E_{S}$,

$$
\begin{aligned}
& a=\left(e,\left(a_{1}, a_{1}\right), \cdots,\left(a_{n}, a_{n}\right)\right), \\
& b=\left(e,\left(b_{1}, b_{1}\right),\left(b_{2}, b_{2}\right), \cdots,\left(b_{n}, b_{n}\right)\right),
\end{aligned}
$$

$a \rho b$ and $a_{k+1} \neq b_{k+1}$ (say, $\left.a_{k+1}>b_{k+1}\right)(k<n)$. If

$$
s=\left(e,\left(a_{1}, b_{1}\right),\left(a_{2}, b_{2}\right), \cdots,\left(a_{n}, b_{n}\right)\right),
$$

$s s^{-1}=a$ and $s^{-1} s=b$ by $[9$, theorem 2.3]. Thus, $a s=s=s b$ and $s^{-1} a=s^{-1}=b s^{-1}$. Since $a<b, \quad b s=b a s=a s=s \quad$ and, similarly, $s^{-1} b=s^{-1}$. Thus, by [2, lemma 1.31], the subsemigroup $\left\langle s, s^{-1}\right\rangle$ generated by $s$ and $s^{-1}$ is the bicyclic semigroup. Since $\rho \mid\left\langle s, s^{-1}\right\rangle$ is not an idempotent separating congruence, $\rho \mid\left\langle s, s^{-1}\right\rangle$ is a group congruence by $[2$, corollary 1.32]. By [2, lemma 1.31] and [9, theorem 2.3], there exists

$c=\left(e,\left(a_{1}, a_{1}\right), \cdots,\left(a_{k}, a_{k}\right),\left(c_{k+1}, c_{k+1}\right),\left(a_{k+2}, a_{k+2}\right), \cdots,\left(a_{n}, a_{n}\right)\right) \in E_{\left\langle s, s^{-1}\right\rangle}$ such that $c_{k+1}-b_{k+1} \geqq 2$. Clearly, each two idempotents of $\left\langle s, s^{-1}\right\rangle$ are $\rho$-equivalent. Let $t=\left(e,\left(a_{1}, a_{1}\right), \cdots,\left(a_{k}, a_{k}\right),(1,0),(0,0), \cdots,(0,0)\right)$. Thus, as above, $\left\langle t, t^{-1}\right\rangle$ is the bicyclic semigroup, and, by [2, lemma 1.31],

$$
E_{\left\langle t, t^{-1}\right\rangle}=\left\{e_{a}=\left(e,\left(a_{1}, a_{1}\right), \cdots,\left(a_{k}, a_{k}\right),(a, a),(0,0), \cdots,(0,0)\right): a \in I^{0}\right\} .
$$


Clearly, $c<e_{\boldsymbol{c}_{k+1}}<e_{c_{k+1}-1}<b$. However, if $x, y, z \in E_{S}, x<y<z$, and $x \rho z$, then $x \rho y$. Hence, since $c \rho b, e_{c_{k+1}} \rho e_{c_{k+1}-1}$. Thus, $\rho \mid\left\langle t, t^{-1}\right\rangle$ is a group congruence on $\left\langle t, t^{-1}\right\rangle$. Therefore, $e_{a} p e_{b}$ for all $a, b \in I^{0}$. Let

$$
g=\left(e,\left(g_{1}, g_{1}\right), \cdots,\left(g_{n}, g_{n}\right)\right) \text { and } h=\left(e,\left(h_{1}, h_{1}\right), \cdots,\left(h_{n}, h_{n}\right)\right)
$$

belong to $E_{S}$ and suppose that $g_{i}=h_{i}(1 \leqq i \leqq k)$. Let $h<g$ and

$$
u=\left(e,\left(g_{1}, a_{1}\right), \cdots,\left(g_{k}, a_{k}\right),\left(g_{k+1}, g_{k+1}\right), \cdots,\left(g_{n}, g_{n}\right)\right) .
$$

Then $u u^{-1}=g$ and

$$
u^{-1} h u=\left(e,\left(a_{1}, a_{1}\right), \cdots,\left(a_{k}, a_{k}\right),\left(i_{k+1}, i_{k+1}\right), \cdots,\left(i_{n}, i_{n}\right)\right)
$$

where $i_{k+1} \in I^{0}, i_{k+1} \geqq h_{k+1}$. Hence,

$$
e_{i_{k+1}+1}<u^{-1} h u<u^{-1} g u=u^{-1}\left(u u^{-1}\right) u=u^{-1} u<e_{0}
$$

and $u^{-1} h u \rho u^{-1} g u$. Thus, $u\left(u^{-1} h u\right) u^{-1} \rho u\left(u^{-1} g u\right) u^{-1}$. However,

$$
u u^{-1} h u u^{-1}=g h g=h \quad \text { and } \quad u u^{-1} g u u^{-1}=g \text {, }
$$

and, hence, $h \rho g$. We have proved that if $g, h \in E_{S}, g \rho h$ if and only if $g_{i}=h_{i}$ for $1 \leqq i \leqq k$ (if $k \geqq 1$ ). Hence, by corollary $1.1, \rho$ is an $\omega^{k}$-bisimple congruence. If $k=0, \rho$ is a group congruence.

REMARK. We thank the referee for the above proof which shortens our original proof.

Remark. In the case $n=1$, we obtain a result of Munn and Reilly $[4$, theorem 1.3]. See [8, theorem 4.1] for another proof of this case.

\section{The maximal group homomorphic image}

In this section, we give a complete determination of the maximal group homomorphic image of an $\omega^{n}$-bisimple semigroup

$$
S=\left(G, C_{n}, \gamma_{1}, \gamma_{2}, \cdots, \gamma_{n}, t_{1}, t_{2}, \cdots, t_{\phi(n)}\right) .
$$

To do this, we must first determine the homomorphisms of $S$ into a group. This determination is based on the homomorphism theory of bisimple inverse semigroups with identity [5]. We also utilize our determination of the maximal group homomorphic image of an $\omega$-bisimple semigroup [8, theorem 3.4].

To determine the homomorphisms of an $\omega^{n}$-bisimple semigroup into a group, we must first determine the homomorphisms of an $\omega^{n}$-right cancellative semigroup into a group.

Theorem 2.1. Let $P=\left(G,\left(I^{0}\right)^{n}, \gamma_{1}, \gamma_{2}, \cdots, \gamma_{n}, t_{1}, t_{2}, \cdots, t_{\phi(n)}\right)$ be an 
$\omega^{n}$-right cancellative semigroup and let $H$ be a group. Let $f$ be a homomorphism of $G$ into $H$ and $z_{1}, z_{2}, \cdots, z_{n} \in H$ such that

$$
\begin{aligned}
z_{l+k} C_{z_{l}} & =t_{\varphi(n-l)+k} f, & & 1 \leqq l \leqq n-1,1 \leqq k \leqq n-l \\
f C_{z_{i}} & =\gamma_{i} f, & & 1 \leqq i \leqq n .
\end{aligned}
$$

Then,

$$
\left(g, n_{1}, n_{2}, \cdots, n_{n}\right) M=g f \prod_{i=n}^{1} z_{i}^{n_{i}}
$$

is a homomorphism of $P$ into $H$ and conversely each such homomorphism is obtained in this fashion. If $f$ is 'onto', $M$ is 'onto'.

Proof. We will not repeat arguments given in the proof of lemma 1.1, but we will use the notation introduced in the proof of lemma 1.1. We let $P^{*} \mid \mathscr{L}^{*}=e^{*}$, the identity of $H$ (notation of [5, theorem 2.3]). We apply $[5$, p. 1119, theorem 2.3]. We first assume the conditions of the theorem and establish [5, p. 1119, Eq. 2.12]. Let $\left(a_{1}, a_{2}, \cdots, a_{n}\right),\left(b_{1}, b_{2}, \cdots, b_{n}\right) \in P / \mathscr{L}$ with $a_{i}=0$ for $1 \leqq i \leqq l-1<n-1$ and $a_{l}>0$. Thus, by [9, equation $1.21]$, (1), and (2), we have

$$
\begin{gathered}
\left(a_{1}, a_{2}, \cdots, a_{n}\right)^{\left(b_{1}, b_{2}, \cdots, b_{n}\right)} f=\left(\prod_{k=0}^{k=n-l-1} t_{\varphi(n-k+1)-k}^{b_{n}} \gamma_{l}^{a_{l}-1} \prod_{i=l+1}^{n} \gamma_{i}^{a_{i}}\right) f \\
=z_{n}^{a_{n}} \cdots z_{l+1}^{a_{l+1}} z_{l}^{a_{l}-1}\left(\prod_{k=0}^{n-l-1} t_{\varphi(n-k)+(n-l-k)}^{b_{n-k}}\right) z_{l}^{-a_{l}+1} z_{l+1}^{-a_{l+1}} \cdots z_{n}^{-a_{n}} \\
=z_{n}^{a_{n}} \cdots z_{l+1}^{a_{l+1}} z_{l}^{a_{l}-1}\left(\prod_{k=0}^{n-l-1} z_{(n-k)}^{b_{n}-k} C_{z_{l}}\right) z_{l}^{-a_{l}+1} z_{l+1}^{-a_{l+1}} \cdots z_{n}^{-a_{n}}
\end{gathered}
$$

Thus, if we let $\left(a_{1}, a_{2}, \cdots, a_{n}\right) h=\prod_{s=n}^{s=1} z_{s}^{a_{s}}$, we obtain the desired equality for this case. The other case is almost immediate. Since

$$
A^{\left(a_{1}, a_{2}, \cdots, a_{n}\right)}=A \gamma_{1}^{a_{1}} \gamma_{2}^{a_{2}} \cdots \gamma_{n}^{a_{n}}(A \in G)
$$

by [9, equation 1.22$]$, utilizing (2), we readily establish [5, p. 1119, Eq. 2.13]. Thus, by [5, p. 1119, Eq. (2.14)], (3) defines a homomorphism of $P$ into $H$. Next, suppose that $M$ is a homomorphism of $P$ into $H$. Let us denote by $u_{i}$ the $n$-tuple with 1 in the $i$-th position and zeroes elsewhere and let $u_{i} h=z_{i} \cdot(1)$ and the fact that $\left(a_{1}, a_{2}, \cdots, a_{n}\right) h=\prod_{i=n}^{1} z_{i}^{a_{i}}$ are consequences of [9, equation 1.21], [5, p. 1119, equation 2.12], and [9, corollary 1.1]. (2) is a consequence of $[9$, equation 1.22$]$ and $[5$, p. 1119 , equation 2.13$]$. By [5, p. 1119, equation 2.14], $M$ is given by (3).

THEOREM 2.2. Let $S=\left(G, C_{n}, \gamma_{1}, \gamma_{2}, \cdots, \gamma_{n}, t_{1}, t_{2}, \cdots, t_{\phi(n)}\right)$ be an $\omega^{n}$-bisimple semigroup and let $H$ be a group. Let $f$ be a homomorphism of $G$ into $H$ and $z_{1}, z_{2}, \cdots, z_{n} \in H$ such that 


$$
\begin{aligned}
z_{l+k} C_{z_{l}} & =t_{\phi(n-l)+k} f, & & 1 \leqq l \leqq n-1,1 \leqq k \leqq n-l \\
f C_{z_{i}} & =\gamma_{i} f, & & 1 \leqq i \leqq n .
\end{aligned}
$$

Then,

$$
\left(g,\left(a_{1}, b_{1}\right), \cdots,\left(a_{n}, b_{n}\right)\right) \Phi=\left(\prod_{i=1}^{n} z_{i}^{-a_{i}}\right)(g f)\left(\prod_{i=n}^{1} z_{i}^{b_{i}}\right)
$$

is a homomorphism of $S$ into $H$ and conversely every such homomorphism obtained in this fashion.

Proof. We will not repeat arguments given in the proof of lemma 1.1, but we will use the notation introduced in the proof of lemma 1.1. Let us assume the conditions of the theorem are valid. Hence, $(3)$ of theorem 2.1 defines a homomorphism $N$ of $P=\left(G,\left(I^{0}\right)^{n}, \gamma_{1}, \gamma_{2}, \cdots, \gamma_{n}, t_{1}, t_{2}, \cdots, t_{q(n)}\right)$ into $H$. Thus, by $[5$, theorem 1.1$]$ and $[1$, p. 548, equation 1.2$]$,

$$
\begin{aligned}
\left(\left(e, a_{1}, a_{2}, \cdots, a_{n}\right),\right. & \left.\left(g, b_{1}, b_{2}, \cdots, b_{n}\right)\right) M \\
& =\left(\left(e, a_{1}, a_{2}, \cdots, a_{n}\right) N\right)^{-1}\left(g, b_{1}, b_{2}, \cdots, b_{n}\right) N
\end{aligned}
$$

defines a homomorphism of $T$ into $H$. Thus, (3), where $\Phi=\delta^{-1} M$, defines a homomorphism of $S$ into $H$.

To establish the converse, let $\Phi$ be a homomorphism of $S$ into $H$. Thus, $M=\delta \Phi$ defines a homomorphism of $T$ into $H$. Hence, $M$ is given by [5, equation 1.14] by virtue of [5, theorem 1.1]. As in the proof of lemma 1.1, we may take $k$ to be the identity of $H$ in [5, equation 1.14]. Thus, by $[1$, p. 548, equation 1.2$]$,

$$
\begin{aligned}
\left(\left(e, a_{1}, a_{2}, \cdots, a_{n}\right),\right. & \left.\left(g, b_{1}, b_{2}, \cdots, b_{n}\right)\right) M \\
& =\left(\left(e, a_{1}, a_{2}, \cdots, a_{n}\right) N\right)^{-1}\left(g, b_{1}, b_{2}, \cdots, b_{n}\right) N
\end{aligned}
$$

where $N$ is a homomorphism of $P$ into $H$. Hence, (1) and (2) are valid, and $\Phi$ is given by (3) by virtue of theorem 2.1 .

We are now in a position to determine the maximal group homomorphic image of an $\omega^{n}$-bisimple semigroup.

If $\sigma$ is an equivalence relation on a set $X$, we let $x_{\sigma}$ denote the equivalence class containing the element $x$ of $X$.

Theorem 2.3. Let $S=\left(G, C_{n}, \gamma_{1}, \gamma_{2}, \cdots, \gamma_{n}, t_{1}, t_{2}, \cdots, t_{\phi(n)}\right)$ be an $\omega^{n}$-bisimple semigroup and lev $e$ be the identity of $G$. If

$$
N=\left\{g \in G \mid g \gamma_{1}^{n}=\text { e for some } n \in I^{\mathbf{0}}\right\},
$$

$N$ is a normal subgroup of $G$. If $(x N) \theta=\left(x \gamma_{1}\right) N, x \in G, \theta$ is an endomorphism of $G / N$. Let $g \rightarrow \bar{g}$ denote the natural homomorphism of $G$ onto $G / N$. Let us define a relation $\sigma$ on $G / N \times\left(I^{0}\right)^{2}$ by the rule

$$
((\bar{g}, a, b),(\bar{h}, c, d)) \in \sigma
$$


if and only if there exists $x, y \in I^{0}$ such that $x+a=y+c, x+b=y+d$, and $\bar{g} \theta^{x}=\bar{h} \theta^{v}$. Then $\sigma$ is an equivalence relation on $G / N \times\left(I^{0}\right)^{2}$. Furthermore, the rule

$$
(\bar{g}, a, b)_{\sigma}(\bar{h}, c, d)_{\sigma}=\left(\bar{g} \theta^{c} \bar{h} \theta^{b}, a+c, b+d\right)_{\sigma}
$$

defines a binary operation on $G / N \times\left(I^{0}\right)^{2} / \sigma=V$ whereby $V$ becomes a group which is the maximal group homomorphic image of $S$. The canonical homomorphism of $S$ onto $V$ is given by

$$
\begin{aligned}
\left(g,\left(n_{1}, m_{1}\right),\left(n_{2}, m_{2}\right), \cdots,\left(n_{n}, m_{n}\right)\right) \zeta \\
=\left(\prod_{s=2}^{n} t_{\varphi(n-1)+(s-1)}^{-n_{s}} \bar{g} \theta \prod_{s=n}^{2} t_{\varphi(n-1)+(s-1)}^{m}, 1+n_{1}, 1+m_{1}\right)_{\sigma} .
\end{aligned}
$$

Proof. Let $T=\left\{\left(g,\left(n_{1}, m_{1}\right),(0,0), \cdots,(0,0)\right): g \in G, n_{1}, m_{1} \in I^{0}\right\}$. By $\left[9\right.$, theorem 2.3], $T$ is an $\omega$-bisimple semigroup $\left(G, C_{1}, \gamma_{1}\right)$. Thus, by [8, theorem 3.4], $N$ is a normal subgroup of $G, \theta$ is an endomorphism of $G / N, \sigma$ is an equivalence relation and $V$ is a group. By a direct calculation, $(\bar{e}, 0,0)_{\sigma}$ is the identity of $V$ and the inverse of $(\bar{g}, a, b)_{\sigma}$ is $\left(\bar{g}^{-1}, b, a\right)_{\sigma}$.

We first employ theorem 2.2 to show that $\zeta$ is a homomorphism of $S$ into $V$. Let us first verify (1) of theorem 2.2. In the notation of that theorem, let $z_{1}=(\bar{e}, 0,1)_{\sigma}$ and $z_{s}=\left(t_{\varphi(n-1)+(s-1)}, 1,1\right)_{\sigma}$ for $s>1$ and let $g f=(\bar{g}, 0,0)_{\sigma}$. We have

$$
(\bar{e}, 0,1)_{\sigma}\left(t_{\varphi(n-1)+k}, 1,1\right)_{\sigma}(\bar{e}, 1,0)_{\sigma}=\left(t_{\varphi(n-1)+k} \theta^{2}, 2,2\right)_{\sigma}=\left(t_{\varphi(n-1)+k}, 0,0\right)_{\sigma},
$$

i.e. $z_{k+1} C_{z_{1}}=t_{q(n-1)+k} f$ for $\mathbf{1} \leqq k \leqq n-1$. Next, we suppose that $l>1$. Thus,

$$
\begin{aligned}
\left(t_{\varphi(n-1)+(l-1)}, 1,1\right)_{\sigma} & \left(t_{\varphi(n-1)+(k+l-1)}, 1,1\right)_{\sigma}\left(t_{\varphi(n-1)+(l-1)}^{-1}, 1,1\right)_{\sigma} \\
& =\left(t_{\varphi(n-1)+(l-1)} t_{\varphi(n-1)+(k+l-1)} t_{\varphi(n-1)+(l-1)}^{-1}, 1,1\right)_{\sigma} .
\end{aligned}
$$

However, we may now employ [9, (2) of theorem 2.3]. Thus

$$
t_{q(n-1)+r} t_{\varphi(n)-i} t_{\varphi(n-1)+r}^{-1}=t_{\varphi(n-r)-i} \gamma_{1}
$$

where $1 \leqq r \leqq n-2$ and $0 \leqq i \leqq n-r-2$. Since $\varphi(n)=\varphi(n-1)+(n-1)$ and $\varphi(n-r)=\varphi(n-r-1)+(n-r-1)$, we have that

$$
t_{\varphi(n-1)+r} t_{\varphi(n-1)+(n-1-i)} t_{\varphi(n-1)+r}^{-1}=t_{\varphi(n-r-1)+(n-r-1-i)} \gamma_{1} .
$$

If we let $r=l-1$ and

$$
i=n-k-l, t_{\varphi(n-1)+(l-1)} t_{\varphi(n-1)+(k+l-1)} t_{\varphi(n-1)+(l-1)}^{-1}=t_{\varphi(n-l)+k} \gamma_{1}
$$

where $2 \leqq l \leqq n-1$ and $1 \leqq k \leqq n-l$. Hence,

$$
\begin{aligned}
\left(t_{\varphi(n-1)+(l-1)} t_{\varphi(n-1)+(k+l-1)} t_{\varphi(n-1)+(l-1)}^{-1}, 1,1\right)_{\sigma} & \left.=\overline{\left(t_{\varphi(n-l)+k} \gamma_{1}\right.}, 1,1\right)_{\sigma} \\
& =\left(\bar{t}_{\varphi(n-l)+k} \theta, 1,1\right)_{\sigma} \\
& =\left(\bar{t}_{\varphi(n-l)+k}, 0,0\right)_{\sigma}
\end{aligned}
$$


i.e. $z_{k+l} C_{z_{1}}=t_{\varphi(n-l)+k} f$ for $1 \leqq l \leqq n-1, \quad 1 \leqq k \leqq n-l$ and we have established (1) of theorem 2.2. We next verify (2) of theorem 2.2. We have

$$
\begin{aligned}
z_{1} g f z_{1}^{-1} & =(\bar{e}, 0,1)_{\sigma}(\bar{g}, 0,0)_{\sigma}(\bar{e}, 1,0)_{\sigma} \\
& =\left(\overline{g \gamma_{1}}, 0,0\right)_{\sigma} \\
& =g \gamma_{1} f .
\end{aligned}
$$

If $s>1$,

$$
\begin{aligned}
z_{s} g f z_{s}^{-1} & =\left(t_{\varphi(n-1)+(s-1)}, 1,1\right)_{\sigma}(\bar{g}, 0,0)_{\sigma}\left(t_{\varphi(n-1)+(s-1)}^{-1}, 1,1\right)_{\sigma} \\
& =\left(t_{\varphi(n-1)+(s-1)} \bar{g} \theta t_{\varphi(n-1)+(s-1)}^{-1}, 1,1\right)_{\sigma} \\
& =\left(t_{\varphi(n-1)+(s-1)} g \gamma_{1} t_{\varphi(n-1)+(s-1)}^{-1}, 1,1\right)_{\sigma} .
\end{aligned}
$$

However, by $[9,(3)$ of theorem 2.3$]$,

$$
\begin{aligned}
\left.\overline{t_{\varphi(n-1)+(s-1)} g \gamma_{1} t_{\varphi(n-1)+(s-1)}^{-1}}, 1,1\right)_{\sigma} & =\left(\overline{g \gamma_{s} \gamma_{1}}, 1,1\right)_{\sigma} \\
& \left.=\left(\overline{g \gamma_{s}}\right) \theta, 1,1\right)_{\sigma} \\
& =\left(\overline{g \gamma_{s}}, 0,0\right)_{\sigma} \\
& =g \gamma_{s} f \text { for } 2 \leqq s \leqq n .
\end{aligned}
$$

Thus, by (3) of theorem 2.2 ,

$$
\left(g,\left(n_{1}, m_{1}\right),\left(n_{2}, m_{2}\right), \cdots,\left(n_{n}, m_{n}\right)\right) \zeta=\prod_{i=1}^{n} z_{i}^{-n_{i}}(g f) \prod_{i=n}^{1} z_{i}^{m_{i}}
$$

is a homomorphism of $S$ into $V$. Thus,

$$
\begin{aligned}
\left(g,\left(n_{1}, m_{1}\right),\left(n_{2}, m_{2}\right), \cdots,\left(n_{n}, m_{n}\right)\right) \zeta=\left(e, n_{1}, 0\right)_{\sigma} \\
\cdot \prod_{s=2}^{n}\left(t_{\varphi(n-1)+(s-1)}^{-n_{1}}, 1,1\right)_{\sigma}(\bar{g}, 0,0)_{\sigma} \prod_{s=n}^{s=2}\left(t_{\Phi(n-1)+(s-1)}^{m_{s}}, 1,1\right)_{\sigma}\left(e, 0, m_{1}\right)_{\sigma} \\
=\left(\prod_{s=2}^{n} t_{\varphi(n-1)+(s-1)}^{-n_{s}} \bar{g} \theta \prod_{s=n}^{2} t_{\varphi(n-1)+(s-1)}^{m_{s}}, n_{1}+1, m_{1}+1\right)_{\sigma} .
\end{aligned}
$$

Since

$$
\begin{aligned}
\left(g,\left(n_{1}, m_{1}\right),(0,0), \cdots,(0,0)\right) \zeta & =\left(\bar{g} \theta, n_{1}+1, m_{1}+1\right)_{\sigma} \\
& =\left(\bar{g}, n_{1}, m_{1}\right)_{\sigma}
\end{aligned}
$$

it is clear that $\zeta$ maps $S$ onto $V$.

We note that $V$ is the maximal group homomorphic image of $T$ under the homomorphism

$$
(g,(a, b),(0,0), \cdots,(0,0)) \Phi=(\bar{g}, a, b)_{\sigma}
$$

by $[8$, theorem 3.4$]$.

Let $\delta$ be a homomorphism of $S$ onto some group $X$. We first show that $\delta \mid T$ is a homomorphism of $T$ onto $X$. If $x \in X,\left(g,\left(n_{1}, m_{1}\right), \cdots,\left(n_{n}, m_{n}\right)\right) \delta=x$ for some $\left(g,\left(n_{1}, m_{1}\right), \cdots,\left(n_{n}, m_{n}\right)\right) \in S$. Thus, by theorem 2.2 , there exists 
$p_{1}, p_{2}, \cdots, p_{n} \in X$ and a homomorphism $f_{1}$ of $G$ into $X$ such that

$$
\begin{aligned}
x & =p_{1}^{-n_{1}} \prod_{i=2}^{n} p_{i}^{-n_{i}} g f_{1} \prod_{i=n}^{2} p_{i}^{m_{i}} p_{1}^{m_{1}} \\
& =p_{1}^{-n_{1}} p_{1}^{-1}\left(\prod_{i=1}^{n-1} t_{\varphi(n-1)+i}^{-n_{i+1}}\right) f_{1} p_{1} g f_{1} p_{1}^{-1}\left(\prod_{i=n-1}^{1} t_{\varphi(n-1)+i}^{m_{i+1}}\right) f_{1} p_{1} p_{1}^{m_{1}} \\
& =p_{1}^{-\left(n_{1}+1\right)}\left(\prod_{i=1}^{n-1} t_{\varphi(n-1)+i}^{-n_{i+1}} g \gamma_{1} \prod_{i=n-1}^{1} t_{\varphi(n-1)+i}^{m_{i+1}}\right) f_{1} p_{1}^{m_{1}+1} \\
& =\left(\left(\prod_{i=1}^{n-1} t_{\varphi(n-1)+i}^{-n_{i+1}} g \gamma_{1} \prod_{i=n-1}^{1} t_{\varphi(n-1)+i}^{m_{i+1}}\right),\left(n_{1}+1, m_{1}+1\right),(0,0), \cdots,(0,0)\right) \delta .
\end{aligned}
$$

Hence there exists a homomorphism $\eta$ of $V$ onto $X$ such that $\Phi_{\eta}=\delta \mid T$. We will show that $\zeta \eta=\delta$, i.e. $H$ is the maximal group homomorphic image of $S$ under the homomorphism $\zeta$. We note that

Hence,

$$
\begin{aligned}
\left(\bar{g}, n_{1}, m_{1}\right)_{\sigma} \eta & =\left(g,\left(n_{1}, m_{1}\right),(0,0), \cdots,(0,0)\right) \Phi_{\eta} \\
& =\left(g,\left(n_{1}, m_{1}\right),(0,0), \cdots,(0,0)\right) \delta .
\end{aligned}
$$

$$
\begin{aligned}
\left(g,\left(n_{1}, m_{1}\right),\right. & \left.\cdots,\left(n_{n}, m_{n}\right)\right) \zeta \eta \\
& =\left(\prod_{k=2}^{n} t_{\varphi(n-1)+(k-1)}^{-n_{k}} \theta \prod_{k=n}^{2} t_{\varphi(n-1)+(k-1)}^{m_{k}}, 1+n_{1}, 1+m_{1}\right)_{\sigma} \eta \\
& =\left(\prod_{k=2}^{n} t_{\varphi(n-1)+(k-1)}^{-n_{k}} g \gamma_{1} \prod_{k=n}^{2} t_{\varphi(n-1)+(k-1)}^{m_{k}}, 1+n_{1}, 1+m_{1}\right)_{\sigma} \eta \\
& =p_{1}^{-\left(n_{1}+1\right)}\left(\prod_{k=2}^{n} t_{\varphi(n-1)+(k-1)}^{-n_{k}} g \gamma_{1} \prod_{k=n}^{k=2} t_{\varphi(n-1)+(k-1)}^{m_{k}}\right) f_{1} p^{m_{1}+1} .
\end{aligned}
$$

Hence, by (2) and (1) of theorem 2.2 , we have

$$
\begin{aligned}
\left(g,\left(n_{1}, m_{1}\right), \cdots,\left(n_{n}, m_{n}\right)\right) \zeta \eta & =p_{1}^{-n_{1}} \prod_{k=2}^{n} p_{k}^{-n_{k}} g f \prod_{k=n}^{2} p_{k}^{m_{k}} p_{1}^{m_{1}} \\
& =\left(g,\left(n_{1}, m_{1}\right),\left(n_{2}, m_{2}\right), \cdots,\left(n_{n}, m_{n}\right)\right) \delta .
\end{aligned}
$$

REMARK. In the case $n=1$, we obtain [8, theorem 3.4].

\section{The idempotent separating congruences}

In this section, we completely determine the idempotent separating congruences of an $\omega^{n}$-bisimple semigroup

$$
S=\left(G, C_{n}, \gamma_{1}, \gamma_{2}, \cdots, \gamma_{n}, t_{1}, t_{2}, \cdots, t_{\varphi(n)}\right)
$$

in terms of the $\gamma_{1}-\gamma_{2} \cdots-\gamma_{n}$ invariant subgroups of $G$ (a normal subgroup $N$ of a group $G$ is $\gamma_{1}-\gamma_{2}-\cdots-\gamma_{n}$ invariant if $N \gamma_{i} \subseteq N$ for $\left.1 \leqq i \leqq n\right)$. 
We utilize a description of the idempotent separating congruences of a bisimple inverse semigroup with identity [7, p. 205, theorem 2]. To do this, we must first determine the right normal divisors of an $\omega^{n}$-right cancellative semigroup.

Let $P$ be a right cancellative semigroup with identity and group of units $U$. A subgroup $V$ of $U$ is called a right normal divisor of $P$ if $a V \subseteq V a$ for all $a \in P$.

Lemma 3.1. Let $P=\left(G,\left(I^{0}\right)^{n}, \gamma_{1}, \gamma_{2}, \cdots, \gamma_{n}, t_{1}, t_{2}, \cdots, t_{\varphi(n)}\right)$ be an $\omega^{n}$-right cancellative semigroup. The right normal divisors of $P$ are the $\gamma_{1}-\gamma_{2} \cdots-\gamma_{n}$ invariant subgroups of $G$.

Proof. Let $(g)_{l}$, with $l \in I^{0}$ and $l \geqq 1$, denote the element of $P$ with $g$ in the first position, 1 in the $l+1 s t$ position and zeroes elsewhere. Let $V$ be a normal divisor of $P$ and let $v \in V$. Hence, if $1 \leqq l \leqq n$, we have, by $[9$, theorem 1.4$]$ that

$$
(e)_{l}(v, 0,0, \cdots, 0)=\left(v \gamma_{l}\right)_{l}=\left(v_{1}, 0,0,0, \cdots 0\right)(e)_{l}=\left(v_{1}\right)_{l}
$$

for some $v_{1} \in V, i e, V$ is $\gamma_{1}-\gamma_{2}-\cdots-\gamma_{n}$ invariant. Conversely, suppose that $V$ is a $\gamma_{1}-\gamma_{2}-\cdots-\gamma_{n}$ invariant subgroup of $G$. Let $v \in V$ and $\left(X, a_{1}, a_{2}, \cdots, a_{n}\right) \in P \quad$ with $a_{l}>0, \quad a_{i}=0 \quad$ for $\quad 1 \leqq i \leqq l-1$, and $1 \leqq l \leqq n-1$. Thus, by [9, theorem 1.4],

$$
\begin{aligned}
\left(X, a_{1}, a_{2}, \cdots, a_{n}\right)(v, 0,0, \cdots, 0) & \\
& =\left(X\left(v \gamma_{l}^{a_{2}} \prod_{i=l+1}^{n} \gamma_{i}^{a_{i}}\right) X^{-1}, 0,0, \cdots, 0\right)\left(X, a_{1}, a_{2}, \cdots, a_{n}\right) .
\end{aligned}
$$

The other case is similar. Hence, $V$ is a right normal divisor of $P$.

Theorem 3.1. Let $S=\left(G, C_{n}, \gamma_{1}, \gamma_{2}, \cdots, \gamma_{n}, t_{1}, t_{2}, \cdots, t_{p(n)}\right)$ be an $\omega^{n}$-bisimple semigroup. There exists a $1-1$ correspondence between the idempotent separating congruences of $S$ and the $\gamma_{1}-\gamma_{2}-\cdots-\gamma_{n}$ invariant subgroups of $G$. If $\rho^{V}$ is the congruence corresponding to the $\gamma_{1}-\gamma_{2}-\cdots-\gamma_{n}$ invariant subgroup $V$,

$$
\rho^{V}\left(g,\left(n_{1}, m_{1}\right), \cdots,\left(n_{n}, m_{n}\right)\right)=\left\{\left(v g,\left(n_{1}, m_{1}\right), \cdots,\left(n_{n}, m_{n}\right)\right): v \in V\right\},
$$

i.e. $\left(g,\left(a_{1}, b_{1}\right), \cdots,\left(a_{n}, b_{n}\right)\right) \rho^{V}\left(h,\left(c_{1}, d_{1}\right), \cdots,\left(c_{n}, d_{n}\right)\right)$ if and only if $a_{i}=c_{i}$, $b_{i}=d_{i}(1 \leqq i \leqq n)$, and $V g=V h$. If $V_{1}, V_{2}$ are $\gamma_{1}-\gamma_{2}-\cdots-\gamma_{n}$ invariant subgroups of $G, V_{\mathbf{1}} \subseteq V_{2}$ if and only if $\rho^{V_{1}} \cong \rho^{V_{2}}$. $\mathscr{H}$ is the maximal idempotent separating congruence of $S$. The idempotent separating congruences of $S$ are precisely the congruences such that the congruence class containing the identity is a group and they are uniquely determined by this class.

Proof. It is a consequence of [9, theorem 2.3] and [7, theorem 2] that $\mathscr{H}$ is the maximal idempotent separating congruence of $S$. The re- 
mainder of the theorem follows from lemma 3.1, [7, theorem 2], and an application of the isomorphism $\delta$ [see proof of lemma 1.1].

REMARK. In the case $n=1$, our result includes a theorem of Munn and Reilly [4, lemma 2.3].

\section{The $\omega^{k}$-bisimple congruences}

In this section, we completely determine the $\omega^{k}$-bisimple congruences $(1 \leqq k<n)$ of an $\omega^{n}$-bisimple semigroup $S=\left(G, C_{n}, \gamma_{1}, \cdots, \gamma_{n}, w_{1}, \cdots, w_{\varphi(n)}\right)$ in terms of certain $\gamma_{1}-\gamma_{2}-\cdots-\gamma_{n}$ invariant subgroups of $G$. We utilize lemma 1.1 (based on the homomorphism theory of [5]) and the theory congruences of an arbitrary inverse semigroup $[3, \mathrm{p} .62$, theorem 7.48$]$. We adopt the definitions of [3, p. $57-$ p.62].

TheOREM 4.1. Let $S=\left(G, C_{n}, \gamma_{1}, \gamma_{2}, \cdots, \gamma_{n}, w_{1}, w_{2}, \cdots, w_{\varphi(n)}\right)$ be an $\omega^{n}$-bisimple semigroup and let $k$ be a positive integer less than $n$. Let $N$ be a $\gamma_{1}-\gamma_{2}-\cdots-\gamma_{n}$ invariant subgroup of $G$ such that $h \gamma_{n} \in N$ implies that $h \in N$.

First, suppose there exists $x \in G$ and a positive integer $\mu$ such that

$$
\begin{aligned}
& x \gamma_{k+1} \in x N \\
& \left(x^{q} h\right) N=\left(h \gamma_{k+1}^{\mu q} x^{q}\right) N \quad \text { for } \quad q \in I^{0} \quad \text { and } \quad h \in G \\
& x \gamma_{l} \in w_{\varphi(n-1)+(k-l+1)}^{\mu} N \quad \text { for } \quad 1 \leqq l \leqq k \text {. }
\end{aligned}
$$

Let

$$
\begin{aligned}
\left.A_{\left(a_{1}, a_{2}\right.}, \cdots, a_{k}\right) & =\left\{\left(g,\left(a_{1}, a_{1}\right), \cdots,\left(a_{k}, a_{k}\right),\left(a_{k+1}, b_{k+1}\right), \cdots,\left(a_{n}, b_{n}\right)\right): a_{i}, b_{i} \in I^{0}\right. \\
& \text { for } k+1 \leqq i \leqq n, a_{k+1}-b_{k+1}=\mu r \text { for some integer } r, g \in G, \text { and } \\
& \left.g \gamma_{k+1} \in N\left(\prod_{s=n-k-1}^{1} w_{\varphi(n-k-1)+s}^{a_{k+s+1}}\right) x^{r}\left(\prod_{s=1}^{n-k-1} w_{\varphi(n-k-1)+s}^{-b_{k+8+1}}\right)\right\} .
\end{aligned}
$$

Then,

$$
A=\left\{A_{\left(a_{1}, a_{2}, \cdots, a_{k}\right)}:\left(a_{1}, a_{2}, \cdots, a_{k}\right) \in\left(I^{0}\right)^{k}\right\}
$$

is a kernel normal system and $\rho_{A}$ is an $\omega^{k}$-bisimple congruence.

We have

$$
\left(g,\left(a_{1}, b_{1}\right), \cdots,\left(a_{n}, b_{n}\right)\right) \rho_{A}\left(h,\left(c_{1}, d_{1}\right), \cdots,\left(c_{n}, d_{n}\right)\right)
$$

if and onily if

$$
\begin{gathered}
a_{s}=c_{s} \text { and } b_{s}=d_{s} \text { for } 1 \leqq s \leqq k \\
\left\{\left(\prod_{s=1}^{n-k-1} w_{\varphi(n-k-1)+s}^{-a_{k+s+1}}\right)\left(g \gamma_{k+1}\right)\left(\prod_{s=n-k-1}^{1} w_{\varphi(n-k-1)+s}^{b_{k+s+1}}\right)\right\} \gamma_{k+1}^{d_{k+1}-\min \left(b_{k+1}, d_{k+1}\right)} \\
\cdot\left\{\prod_{s=1}^{n-k-1}\left(w_{\varphi(n-k-1)+s}^{-d_{k+s+1}}\right)\left(h^{-1} \gamma_{k+1}\right)\left(\prod_{s=n-k-1}^{1} w_{\varphi(n-k-1)+s}^{c_{k+s+1}}\right)\right\} \gamma_{k+1}^{b_{k+1}-\min \left(b_{k+1}, d_{k+1}\right)} \in N x^{r}
\end{gathered}
$$


where

Secondly, let

$$
\mu r=\left(a_{k+1}-b_{k+1}\right)+\left(d_{k+1}-c_{k+1}\right), r \in I .
$$

$$
\begin{aligned}
A_{\left(a_{1}, a_{2}, \cdots, a_{k}\right)}^{*} & =\left\{\left(g,\left(a_{1}, a_{1}\right), \cdots,\left(a_{k}, a_{k}\right),\left(a_{k+1}, a_{k+1}\right),\left(a_{k+2}, b_{k+2}\right), \cdots,\left(a_{n}, b_{n}\right)\right)\right. \\
& : a_{i}, b_{i} \in I^{0} \text { for } k+1 \leqq i \leqq n, g \in G, \text { and } \\
& \left.g \gamma_{k+1} \in N\left(\prod_{n-k-1}^{s=1} w_{\Phi(n-k-1)+s}^{a_{k+s+1}}\right)\left(\prod_{s=1}^{n-k-1} w_{\Phi(n-k-1)+\varepsilon}^{-b_{k+k+1}}\right)\right\} .
\end{aligned}
$$

Then,

$$
A^{*}=\left\{A_{\left(a_{1}, \cdots, a_{k}\right.}^{*}:\left(a_{1}, \cdots, a_{k}\right) \in\left(I^{0}\right)^{k}\right\}
$$

is a kernel normal system and $\rho_{A^{*}}$ is an $\omega^{k}$-bisimple congruence.

We have

$$
\left(g,\left(a_{1}, b_{1}\right), \cdots,\left(a_{n}, b_{n}\right)\right) \rho_{A^{*}}\left(h,\left(c_{1}, d_{1}\right), \cdots,\left(c_{n}, d_{n}\right)\right)
$$

if and only if

$$
\begin{gathered}
a_{s}=c_{s} \text { and } b_{s}=d_{s} \text { for } 1 \leqq s \leqq k \\
\left(a_{k+1}-b_{k+1}\right)+\left(d_{k+1}-c_{k+1}\right)=0 \\
\left\{\left(\prod_{s=1}^{n-k-1} w_{\varphi(n-k-1)+s}^{-a_{k+s+1}}\right)\left(g \gamma_{k+1}\right)\left(\prod_{n-k-1}^{s=1} w_{\varphi(n-k-1)+s}^{b_{k+1}}\right)\right\} \gamma_{k+1}^{d_{k+1}}-\min \left(d_{k+1}, b_{k+1}\right) \\
\cdot\left\{\left(\prod_{s=1}^{n-k-1} w_{\varphi(n-k-1)+s}^{-d_{k+s+1}}\right)\left(h^{-1} \gamma_{k+1}\right)\left(\prod_{s=n-k-1}^{1} w_{\varphi(n-k-1)+s}^{c_{k+k}}\right)\right\} \gamma_{k+1}^{b_{k+1}-\min \left(d_{k+1}, b_{k+1}\right)} \in N .
\end{gathered}
$$

In both the above cases, if $n=k+1$, any factor involving ' $\Pi$ ' is replaced by $e$, the identity of $G$.

Conversely, every $\omega^{k}$-bisimple congruence of $S$ is obtained in one of the above fashions.

Proof. Let $\rho$ be an $\omega^{k}$-bisimple congruence on $S$ with $k<n$ and let $K_{\rho}$ be the kernel of $\rho$. Thus, $\rho=\theta \circ \theta^{-1}$ where $\theta$ is a homomorphism of $S$ into $S / \rho$. We will use the notation of lemma 1.1 with

$$
S / \rho=\left(G^{*}, \alpha_{1}, \alpha_{2}, \cdots, \alpha_{k}, t_{1}, t_{2}, \cdots, t_{q(k)}\right) .
$$

By lemma 1.1 (definition of $\theta$ ),

$$
\left(g,\left(a_{1}, b_{1}\right),\left(a_{2}, b_{2}\right), \cdots,\left(a_{k}, b_{k}\right),\left(a_{k+1}, b_{k+1}\right), \cdots,\left(a_{n}, b_{n}\right)\right) \in K_{\rho}
$$

if and only if $a_{i}=b_{i}$ for $1 \leqq i \leqq k$ and

$$
g f=\left(\prod_{s=n-k-1}^{s=1} z_{k+s+1}^{a_{k+1+1}}\right) z_{k+1}^{a_{k+1}-b_{k+1}}\left(\prod_{s=1}^{n-k-1} z_{k+1+s}^{-b_{k+s+1}}\right)
$$

if $n>k+1$. ( $g f=z_{n}^{a_{n}-b_{n}}$ if $n=k+1$.) Equivalently, by virtue of (3) and (5) of lemma 1.1, if $n>k+1$, 


$$
*_{z_{k+1}} g f z_{k+1}^{-1}=\left(\prod_{s=n-k-1}^{s=1} w_{\varphi(n-k-1)+s}^{a_{k+s+1}}\right) f z_{k+1}^{a_{k+1}-b_{k+1}}\left(\prod_{s=1}^{s=n-k-1} w_{(n-k-1)+s}^{-b_{k+s+1}}\right) f=g \gamma_{k+1} f
$$

or, if $n=k+1,{ }^{* *} g \gamma_{n} f=z_{n}^{a_{n}-b_{n}}$ by virtue of (5) of lemma 1.1.

Suppose there exists a positive integer $m$ such that $z_{k+1}^{m} \in G f$. Let $\mu$ be the smallest such positive integer. Hence,

$$
G f \cap\left(z_{k+1}\right)=\left\{z_{k+1}^{\mu p}: p \in I, \text { the integers }\right\} .
$$

Thus, if $x f=z_{k+1}^{\mu}$, it is easily seen that '*' is equivalent to the statement

$$
g \gamma_{k+1} \in N\left(\prod_{s=n-k-1}^{s=1} w_{\varphi(n+k-1)+s}^{a_{k+s+1}}\right) x^{n}\left(\prod_{s=1}^{n-k-1} w_{\varphi(n-k-1)+s}^{-b_{k++1}}\right)
$$

where $\mu r=a_{k+1}-b_{k+1}, r \in I$, and where $N$ is the kernel of $f$ while '**' is equivalent to

$$
g \gamma_{k+1} \in N x^{\tau} .
$$

By (5) of lemma 1.1, $h \gamma_{n} \in N$ implies $h \in N$ and (1) and (2) are valid. By (4) and (5) of lemma $1.1, N$ is $a \gamma_{1}-\gamma_{2}-\cdots-\gamma_{n}$ invariant subgroup of $G$. By (2) and (4) of lemma 1.1, (3) is valid.

Next, suppose that $\left(z_{k+1}\right) \cap G f=e^{*}$, the identity of $G^{*}$. Thus, * is equivalent to

$$
g \gamma_{k+1} \in N\left(\prod_{s=n-k-1}^{s=1} w_{\varphi(n-k-1)+s}^{a_{k+s+1}}\right)\left(\prod_{s=1}^{s=n-k-1} w_{\varphi(n-k-1)+8}^{-b_{k+s+1}}\right)
$$

and $a_{k+1}=b_{k+1}$ while $(* *)$ is equivalent to $g \gamma_{n} \in N$.

The verification that $A$ and $A^{*}$ are kernel normal systems, subject to the conditions of the theorem, is long and tedious. Thus, we will content ourselves with sketching a few sample cases. We will utilize the multiplication given in [9, theorem 2.3] without explicit mention.

We first note that

$$
A \prod_{i=l+1}^{n} \gamma_{i}^{b_{i}} \gamma_{k+1}=\left(\prod_{s=n-k-1}^{l-k} w_{\Phi(n-k-1)+s}^{b_{k+k}}\right)\left(A \gamma_{k+1}\right)\left(\prod_{s=l-k}^{n-k-1} w_{\varphi(n-k-1)+s}^{-b_{k+s+1}}\right)
$$

for $A \in G$ and $1 \geqq k+1$ by $[9,(3)$ of theorem 2.3$]$ and

$$
\begin{aligned}
& \left(\prod_{s=0}^{s=n-l-1} w_{\varphi(n-l+1)-8}^{d_{n-8}}\right) \gamma_{k+1}=w_{\varphi(n-k-1)+(l-k-1)}\left(\prod_{s=n-k-1}^{l-k} w_{\varphi(n-k-1)+8}^{d_{k(s+1)}}\right) \\
& w_{\varphi(n-k-1)+(l-k-1)}^{-1} \text { for } n>l>k+1 \text { and } k+1 \leqq n-2
\end{aligned}
$$

by $[9,(2)$ of theorem 2.3$]$. Let

$$
\begin{aligned}
\xi & =\left(g,\left(a_{1}, a_{1}\right), \cdots,\left(a_{k}, a_{k}\right),\left(a_{k+1}, b_{k+1}\right), \cdots,\left(a_{n}, b_{n}\right)\right), \eta \\
& =\left(h,\left(a_{1}, a_{1}\right), \cdots,\left(a_{k}, a_{k}\right),\left(c_{k+1}, d_{k+1}\right), \cdots,\left(c_{n}, d_{n}\right)\right) \in A_{\left(a_{1}, a_{2}, \cdots, a_{k}\right)} .
\end{aligned}
$$

If, for example, $k+1<n$ and $b_{k+1}>c_{k+1}$, then $\xi \eta \in A_{\left(a_{1}, a_{2}, \cdots, a_{k}\right)}$, by virtue 
of (1), (9), and the fact that $N$ is a $\gamma_{k+1}$-invariant subgroup of $G$. Clearly, $A_{\left(a_{1}, a_{2}, \cdots, a_{n}\right)}$ is closed under the operation of taking inverses and hence $[3, \mathrm{p} .60, K 1]$ is verified in this case.

Let

and let

$$
a=\left(h,\left(p_{1}, q_{1}\right), \cdots,\left(p_{n}, q_{n}\right)\right)
$$

$$
b=\left(g,\left(a_{1}, a_{1}\right), \cdots,\left(a_{k}, a_{k}\right),\left(a_{k+1}, b_{k+1}\right), \cdots,\left(a_{n}, b_{n}\right)\right) \in A_{\left(a_{1}, a_{2}, \cdots, a_{k}\right)} .
$$

Suppose that $a_{s}=q_{s}$ for $1 \leqq s \leqq n-1$ and $q_{n}>a_{n}$ while $b_{s}=q_{s}$ for $k+1 \leqq s \leqq l-1$ where $k+1<l<n$ and $b_{l}>q_{l}$. Thus, $a b a^{-1} \in A_{\left(p_{1}, p_{2}, \cdots, p_{k}\right)}$ by virtue of (9), (10), $[9$, theorem $2.3,(3)]$, and the fact that $N$ is an invariant subgroup of $G$. If $q_{s}=a_{8}$ for $1 \leqq s \leqq n-1, q_{n}<a_{n}$, and $k+1=n$ with $b_{n}>q_{n}, a b a^{-1} \in A_{\left(p_{1}, p_{2}, \cdots, p_{k}\right)}$ by (2). If $q_{s}=a_{s}$ for $1 \leqq s \leqq l-1$ where $1 \leqq l \leqq k<n$ and $q_{l}>a_{l}, a b a^{-1} \in A_{\left(p_{1}, p_{2}, \cdots, p_{k}\right)}$ by [9, theorem 2.3, (2) and (3)], (3) and the fact that $N$ is a $\gamma_{1}-\gamma_{2} \cdots \cdots \gamma_{n}$ invariant subgroup of $G$. Hence, [3, p. 60, condition $K 4]$ is established in these cases.

Let

and

$$
a=\left(g,\left(a_{1}, a_{1}\right), \cdots,\left(a_{k}, a_{k}\right),\left(a_{k+1}, b_{k+1}\right), \cdots,\left(a_{n}, b_{n}\right)\right)
$$

$$
b=\left(h,\left(c_{1}, d_{1}\right), \cdots,\left(c_{k}, d_{k}\right), \cdots,\left(c_{n}, d_{n}\right)\right)
$$

and suppose that $a, a b$, and $b b^{-1} \in A_{\left(a_{1}, a_{2}, \cdots, a_{k}\right)}$. Hence, in the case $k+\mathrm{I}=n$ and $b_{n}>c_{n}, b \in A_{\left(a_{1}, a_{2}, \cdots, a_{k}\right)}$ by (I) and the condition ' $h \gamma_{n} \in N$ implies that $h \in N^{\prime}$. Hence, [3, p. 60, condition K5] is valid in this case.

The explicit determination of $\rho_{A}$ and $\rho_{A^{*}}$ involves [3, p. 60, equation 1] and the calculations are of a similar character to the above.

REMARK. If $k+1=n$, (5) may be replaced by

$$
g \gamma_{n}^{d_{n}-\min \left(b_{n}, d_{n}\right)} h^{-1} \gamma_{n}^{b_{n}-\min \left(b_{n}, d_{n}\right)} \in N x^{r}
$$

and (8) may be replaced by

$$
\lg \gamma_{n}^{i_{n}-\min \left(b_{n}, d_{n}\right)} h^{-1} \gamma_{n}^{b_{n}-\min \left(b_{n}, d_{n}\right)} \in N .
$$

These statements follow since $h \gamma_{n} \in N$ implies that $h \in N$, and (1) is valid.

Remark. We thank Mr. John Hogan for aiding us in verifying that $A$ is a kernel normal system.

We will state theorem 3.1 in the case $n=2$ and $k=1$ since this represents the simplest possible case.

Theorem 4.2. Let $S=\left(C, C_{2}, \gamma_{1}, \gamma_{2}, w_{1}\right)$ be an $\omega^{2}$-bisimple semigroup and let $N$ be a $\gamma_{1}-\gamma_{2}$ invariant subgroup of $G$ such that $h \gamma_{2} \in N$ implies that $h \in N$. 
First suppose there exists $x \in G$ and a positive integer $\mu$ such that

$$
\begin{aligned}
& x \gamma_{2} \in x N \\
& \left(x^{q} h\right) N=\left(h \gamma_{2}^{\mu q} x^{q}\right) N \text { for } q \in I^{0} \text { and } h \in G \\
& x \gamma_{1} \in w_{1}^{\mu} N .
\end{aligned}
$$

Let

$A_{a_{1}}=\left\{\left(N x^{r},\left(a_{1}, a_{1}\right),\left(a_{2}, b_{2}\right)\right): a_{2}, b_{2} \in I^{0}, a_{2}-b_{2}=\mu r\right.$ for some integer $\left.r\right\}$.

Then, $A=\left\{A_{a_{1}}: a_{1} \in I^{0}\right\}$ is a kernel normal system and $\rho_{A}$ is an $\omega$-bisimple congruence.

We have

if and only if

$$
\left(g,\left(a_{1}, b_{1}\right),\left(a_{2}, b_{2}\right)\right) \rho_{A}\left(h,\left(c_{1}, d_{1}\right),\left(c_{2}, d_{2}\right)\right)
$$

$$
\begin{aligned}
& a_{1}=c_{1} \text { and } b_{1}=d_{1} \\
& g \gamma_{2}^{d_{2}-\min \left(b_{2}, d_{2}\right)} h^{-1} \gamma_{2}^{b_{2}-\min \left(b_{2}, d_{2}\right)} \in N x^{r}
\end{aligned}
$$

where

Secondly, let

$$
\mu r=\left(a_{2}-b_{2}\right)+\left(d_{2}-c_{2}\right), r \in I
$$

$$
A_{a_{1}}^{*}=\left\{\left(N,\left(a_{1}, a_{1}\right),\left(a_{2}, a_{2}\right)\right): a_{2} \in I^{0}\right\} .
$$

Then, $A^{*}=\left\{A_{a_{1}}^{*}: a_{1} \in I^{0}\right\}$ is a kernel normal system and $\rho_{A^{*}}$ is an $\omega$-bisimple congruence.

We have

$$
\left(g,\left(a_{1}, b_{1}\right),\left(a_{2}, b_{2}\right)\right) \rho_{A^{*}}\left(h,\left(c_{1}, d_{1}\right),\left(c_{2}, d_{2}\right)\right)
$$

if and only if

$$
\begin{aligned}
& a_{1}=c_{1} \quad \text { and } \quad b_{1}=d_{1} \\
& a_{2}-b_{2}=c_{2}-d_{2} \\
& g \gamma_{2}^{d_{2}-\min \left(b_{2}, d_{2}\right)} h^{-1} \gamma_{2}^{b_{2}-\min \left(b_{2}, d_{2}\right)} \in N .
\end{aligned}
$$

Conversely, every w-bisimple congruence of $S$ is obtained in one of the above fashions.

REMARK. In [6], we determined the structure of $\omega^{2}$-bisimple semigroups whose structure group is of a type that includes all finite (non-trivial) simple groups, the rationals under addition, and groups of type $p^{\infty}$. The theorems of this paper will specialize to $\omega^{2}$-bisimple semigroups of this type. They will also specialize to this type of $\omega^{n}$-bisimple semigroups.

Added In Proof. A converse has been obtained for lemma 1.1. This will be given elsewhere [10]. 


\section{References}

[1] A. H. Clifford, 'A class of $d$-simple semigroups', Amer. J. Math. 75 (1953), 547-556.

[2] A. H. Clifford and G. B. Preston, The algebraic theory of semigroups, Vol. 1, (Math. Surveys of the American Math. Soc. 7, Providence, R.I., 1961).

[3] A. H. Clifford and G. B. Preston, The algebraic theory of semigroups, Vol. 2, (Math. Surveys of the American Math. Soc. 7, Providence, R.I., 1967).

[4] W. D. Munn and N. R. Reilly, 'Congruences on a bisimple $\omega$-semigroup', Proc. Glasgow Math. Assoc. 7 (1966), $184-192$.

[5] R. J. Warne, 'Homomorphisms of $d$-simple inverse semigroups with identity', Pacific J. Math. 14 (1964), 1111-1122.

[6] R. J. Warne, 'A class of bisimple inverse semigroups', Pacific J. Math., 18 (1966), $563-577$.

[7] R. J. Warne, 'The idempotent separating congruences of a bisimple inverse semigroup with identity', Publicationes Mathematicae 13 (1966), 203-206.

[8] R. J. Warne, 'I-bisimple semigroups', Trans. Amer. Math. Soc., 130 (1968), 367-386.

[9] R. J. Warne, 'Bisimple inverse semigroups mod groups', Duke Math. J. 34 (1967), $787-812$.

[10] R. J. Warne, ' $\omega^{n} I$-bisimple semigroups', to appear.

[11] R. J. Warne, 'Congruences on $L_{n}$-bisimple semigroups', Notices AMS, 14 (1967), 405.

West Virginia University

Morgantown, West Virginia 